\title{
A CASE REPORT OF KAWASAKI DISEASE SHOCK SYNDROME IN A YOUNG PATIENT
}

Straka Kalliopi, Krontsi Alexandra, Paraschou Dimitra, Poulos Panagiotis, Barbaressou Charikleia

Paediatric Intensive Care Unit, Aghia Sophia Children's Hospital, Athens, Greece

picu@paidon-agiasophia.gr

Background and aims
Kawasaki disease (KD) is an acute systemic
vasculitis of unknown aetiology involving medium-
sized arteries, including the coronaries.
Aneurysms develop during the chronic phase.
Although it is known that myocarditis and heart $71 \frac{1}{2}$ year-old boy with
failure can occur, Kawasaki disease Shock
Syndrome (KDSS) has been recently described and
it's possibly multifactorial.

\section{Results}

The patient was admitted to the paediatric clinic with the provisional diagnosis of Kawasaki Disease, fulfilling several criteria with elevated inflammation markers. Initial ECHOs were normal. He received several courses of antibiotics without showing any improvement.

On Day 6 of fever, he presented with respiratory distress, grunting and signs of poor perfusion. Initial CXR showed enlarged heart with pulmonary oedema. Repeated ECHO revealed reduced contractility with mitral and tricuspid regurgitation without pericardial effusion or aneurysms and shortly, the patient developed hemodynamic instability with increased serum troponin.

The differential diagnosis included septic shock, toxic shock syndrome, Stevens Johnson's syndrome, rheumatic diseases (SJRA), scarlet fever and viral myocarditis. He was admitted to PICU requiring resuscitation, volume expansion, diuretics and cardiovascular support with inotropes/vasopressors but remained SVIA on mask with FiO2 $35 \%$ without need for mechanical ventilatory support.

Treatment for KD was promptly started (aspirin and immunoglobulin). All tests for infections, rheumatic and autoimmune diseases were negative. The patient required 5 days of inotropic support with improvement of his cardiac function without formation of aneurysms.

\section{Conclusions}

-KDSS affects $7 \%$ of patients with KD during the acute phase and it can lead to cardiogenic shock.

-Possible mechanisms include increased release of proinflammatory cytokines and VEGF \& capillary leak syndrome.

- Early treatment and follow-up ECHOs are essential in order to avoid longterm cardiac complications and formation of aneurysms. 